\title{
Multi-jet correlations and colour coherence phenomena
}

\author{
Jason S.H. Lee ${ }^{1, a}$ on behalf of the CMS Collaboration \\ ${ }^{1}$ University of Seoul, South Korea
}

\begin{abstract}
In multi-jet events, colour coherence was shown to play a important role in the topology of these events. In modern Monte Carlo generators, the colour coherence effect has become an inherent feature, such that the colour coherence effect can no longer be disentangled in a simple manner. This study looks at the multi-jet correlations by comparing Monte Carlo generators in Parton Shower dominant and Matrix Element dominant regions.
\end{abstract}

\section{Introduction}

When a quark pair is produced, one of the jets can radiate a gluon. Due to quantum chromodynamics (QCD), in the fragmentation phase, the outgoing partons interact with each other. This phenomena was first described by the LUND string model [1], predicting a suppression of particle production in the region between the two quark jets due to destructive interference and an enhancement of particle production in the region between the quark and gluon jets. This colour coherence effect was initially observed in $e^{+} e^{-}$collisions by several experiments at $e^{+} e^{-}$colliders (PETRA, PEP and LEP), leading to the discovery of the gluon.

In hadron collisions, constituents are also coloured making the three jets colour connected to the proton constituents. The Tevatron experiments showed that the variable ' $\beta$ ', the ratio of the difference in pseudorapidity over the difference in azimuthal angle between the second and third jet, is sensitive to colour coherence [2]. The second jet is the jet with the second highest transverse momentum $\left(p_{T}\right)$ and the third jet is most likely to have been the radiated gluon. They showed good agreement with data for parton shower Monte Carlo (MC) generators with the colour coherence effects implemented via angular ordering. They tuned PYTHIA [3] with the angular ordering on and off to highlight this effect.

Section 2 summaries the some of the QCD jets measurement, from the CMS collaboration [4] at the CERN LHC, which are directly and indirectly affected by the colour coherence effect.

This has led to the colour coherence effect becoming an inherent feature of modern MC generators, such that the colour coherence effect can no longer be disentangled in a simple manner. To study the multi-jet correlations, it was suggested by the PYTHIA authors that a more practical test on MC generators would be to test the Parton Shower (PS) dominant and Matrix Element (ME) dominant regions. Section 3 compares four MC generators, one with only PS calculations, one with only ME calculations, and two with both PS and ME calculations.

ae-mail: jason.lee@cern.ch 


\section{Jet Measurement by CMS}

CMS uses particle flow objects as inputs for the anti-kt jet clustering algorithm [5], with the radius parameter set to $\mathrm{R}=0.5$ in Run1, $\mathrm{R}=0.4$ for Run2.

The di-jet azimuthal decorrelation is sensitive to the radiation of additional jets as measured in Run1 in [6]. In the region where the di-jet azimuthal separation is large, $\Delta \phi_{d i-j e t}>2 \pi / 3$, it was shown to be well modelled by the MC generators. However, for the region where the di-jet azimuthal separation is small, $\Delta \phi_{d i-j e t} \approx \pi / 2$, differences started to show up between data and MC as the azimuthal separation decreased. Here, smaller azimuthal separation is due to the production of additional jets and this region is indirectly sensitive to colour coherence.

In the Run1 measurement of the jet charge [7], the jet charge is measured in three directions, in the direction of the transverse momentum, longitudinal to jet axis and transverse to jet axis. A ' $K$ ' factor was introduced to give more weight to low momentum tracks within the jet for small ' $K$ '. The jet charge defined in the direction transverse to the jet axis together with a small ' $K$ ' would be most sensitive to angular correlation effects like colour coherence. And, unsurprisingly, this region showed the biggest discrepancies between data and MC.

Similar to the Tevatron measurement, CMS produced a colour coherence result from Run1 data [8] using the same ' $\beta$ ' variable. The leading order MC generators were shown to have a poor description of the data in forward region. And similarly to the Tevatron measurement, a comparison to data with colour coherence effects turned on and off was shown. Turning on the colour coherence effects with PYTHIA shows better agreement with data compared to the case when colour coherence is turned off. Currently, only a very old version of PYTHIA can turn off colour coherence effects.

\section{Parton Shower vs Matrix Element}

As the colour coherence effect became an inherent feature of MC generators, in the newer MC generators, the colour coherence effect can no longer be disentangled in a simple manner. It was suggested that a more practical test on MC generators would be to test the performance in a Parton Shower (PS) dominant and a Matrix Element (ME) dominant regions. This study used three MC generators, PYTHIA8[3], MADGRAPH[9] and POWHEG BOX[10-12], to make three jet events with only PS, only ME and both ME + PS calculations. PYTHIA8 (version 8.212) is the standard tool for the generation of events in high-energy collisions which includes soft and hard QCD processes and hard QCD processes using standard $2 \rightarrow 2$ processes. Initial state and final state radiations are implemented by parton shower (PS) models which is based on dipole style $p_{T}$-ordered evolution. PYTHIA8 was generated using the latest CUETP8M1 tune, which is based on Monash with NNPDF2.34LO parton distribution function. Both MADGRAPH (version 5.2.4) and POWHEG (version 2) are ME based generator and both using the PYTHIA8 for the PS For MADGRAPH, the QCD processes $2 \rightarrow 3$ was done at leading order (LO) calculation, while POWHEG is next leading order (NLO). Three jet events where also generated using POWHEG only, with PS turned off in PYTHIA8, so that the events would only be generated with ME calculations. Four samples were made to study the PS dominant and ME dominant regions:

- PYTHIA8 (PS only)

- POWHEG + PYTHIA8 without PS (ME only)

- $\mathrm{POWHEG}+\mathrm{PYTHIA8}$ with PS (ME + PS)

- MADGRAPH + PYTHIA8 (ME + PS) 

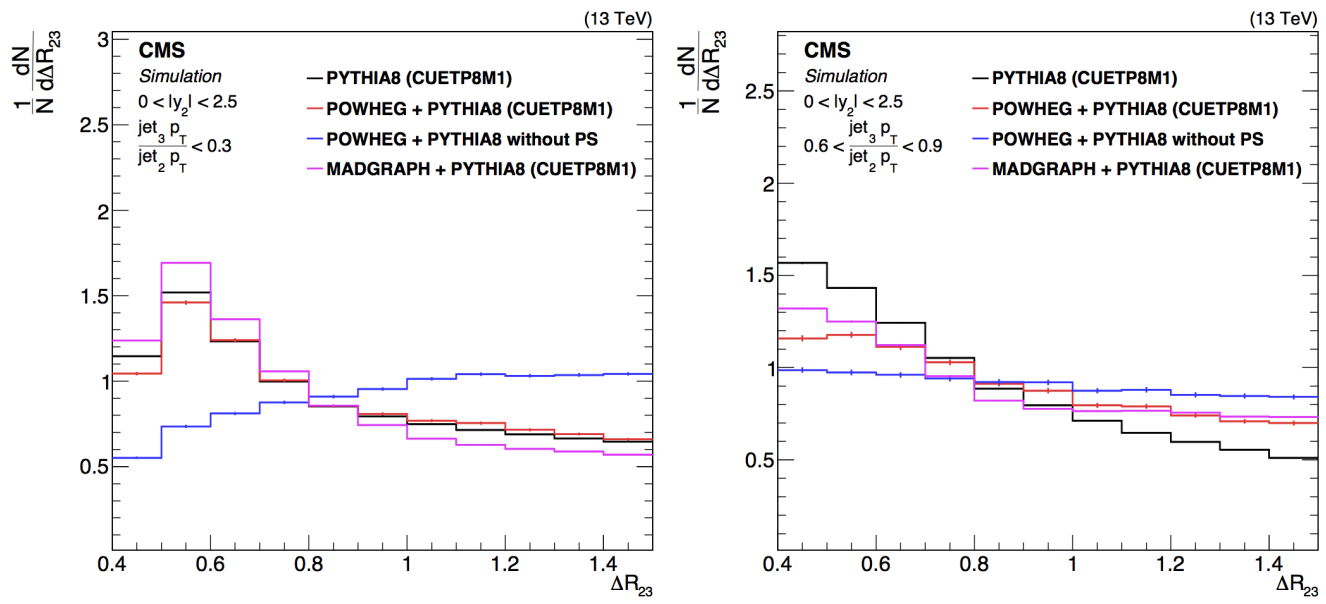

Figure 1. $\Delta R_{23}$ distributions for $p_{T 3} / p_{T 2}<0.3$ PS dominant (left) and high $0.6<p_{T 3} / p_{T 2}<0.9 \mathrm{ME}$ dominant (right) regions. PYTHIA8 is PS only, POWHEG + PYTHIA8 without PS is ME only and both POWHEG + PYTHIA8 and MADGRAPH + PYTHIA8 have ME + PS.

This MC study looked at events with only three jets, all with $p_{T}>30 \mathrm{GeV}$ and $\eta<2.5$. A much higher $p_{T}$ requirement on the leading jet of $510 \mathrm{GeV}$ was used so that it can be triggered in real CMS data. Two variables were used to separate and study the PS dominant and ME dominant regions. The angular separation between the second and third $p_{T}$ ordered jets, $\Delta R_{23}=\sqrt{(\Delta \eta)^{2}+(\Delta \phi)^{2}}$, where $\Delta \eta=\eta_{3}-\eta_{2}$ and $\Delta \phi=\phi_{3}-\phi_{2}$. The second variable is the $p_{T}$ ratio of the third jet over the second jet, $p_{T 3} / p_{T 2}$. A soft third jet should be PS dominated, while a hard third jet should be ME dominated. Soft third jet will have a low $p_{T 3} / p_{T 2}$ and small angular separation $\Delta R_{23}$, while a hard third jet will have $p_{T 3} / p_{T 2}$ near 1 and larger angular separation $\Delta R_{23}$.

Figure 1 shows the $\Delta R_{23}$ distributions for low $p_{T 3} / p_{T 2}<0.3$ and high $0.6<p_{T 3} / p_{T 2}<0.9$ events. In the low $p_{T 3} / p_{T 2}$ region, the third jet is soft and PS is expected to be dominant. POWHEG + PYTHIA8 without PS is ME only and the distribution is very different to all the other samples which have PS. PYTHIA8 agrees well with POWHEG + PYTHIA8 and MADGRAPH + PYTHIA8, even though the latter two also have additional ME contributions. In the high $p_{T 3} / p_{T 2}$ region, the third jet is hard and ME is expected to be dominant. Although it isn't as striking, it is clearly visible that PYTHIA8 isn't as flat as the other samples with ME. This effect is greater at high $\Delta R_{23}$, where POWHEG + PYTHIA8 and MADGRAPH + PYTHIA8 are close to POWHEG + PYTHIA8 without PS.

Figure 2 shows the $p_{T 3} / p_{T 2}$ distributions for small $0.4<\Delta R_{23}<1.0$ and large $1.0<\Delta R_{23}<1.5$ events. In the small $\Delta R_{23}$ region, the third jet has a small angular separation from the second jet and PS is expected to be dominant. PYTHIA8 agrees very well with MADGRAPH + PYTHIA8, while both POWHEG samples seem to be different. In the large $\Delta R_{23}$ region, the third jet has a large angular separation from the second jet and ME is expected to be dominant. PYTHIA8 seems to disagree with all the others, with POWHEG + PYTHIA8 and MADGRAPH + PYTHIA8 closer to POWHEG + PYTHIA8 without PS. 

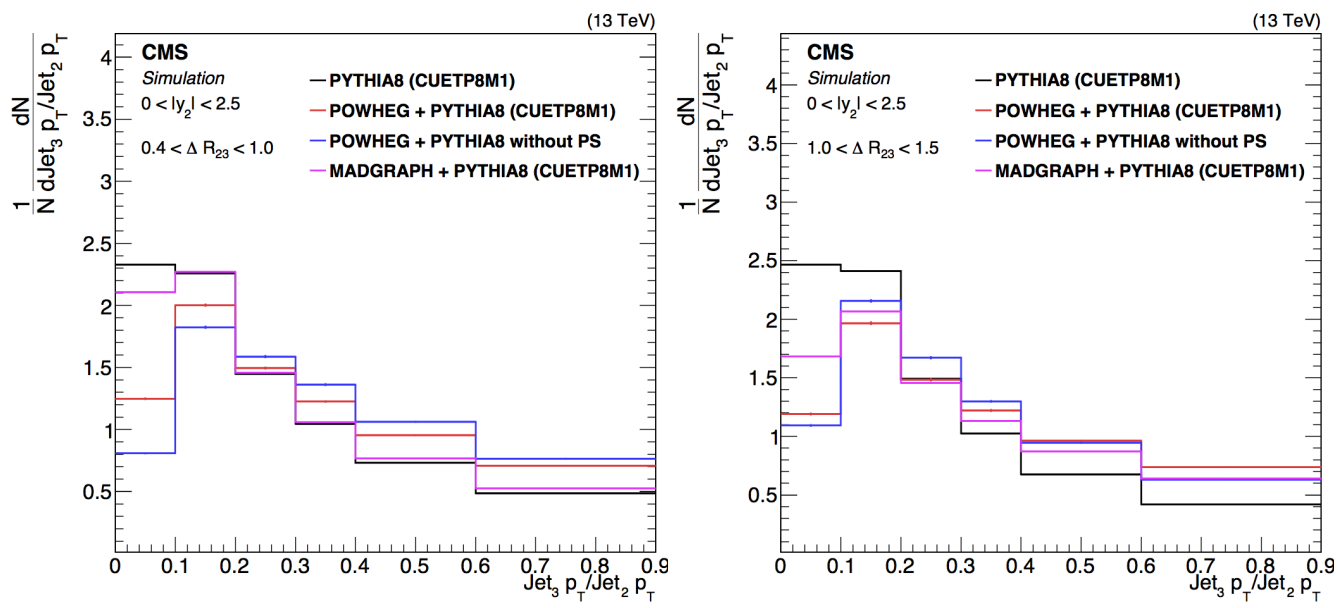

Figure 2. $p_{T 3} / p_{T 2}$ distributions for small $0.4<\Delta R_{23}<1.0$ PS dominant (left) and large $1.0<\Delta R_{23}<1.5$ ME dominant (right) regions. PYTHIA8 is PS only, POWHEG + PYTHIA8 without PS is ME only and both POWHEG + PYTHIA8 and MADGRAPH + PYTHIA8 have ME + PS.

\section{Summary}

As shown by the CMS measurements on jets, multi-jet correlations like colour coherence still needs to be understood better. These results show that the three jet events can be separated into ME and PS dominated regions. Further optimisations could be done to separate the ME and PS even further. Comparisons to data by looking at these regions will make it much easier to understand where the generators are poorly performing.

\section{References}

[1] B. Andersson, G. Gustafson, B. Soderberg, Z. Phys. C20, 317 (1983)

[2] B. Abbott et al. (D0), Phys. Lett. B414, 419 (1997), hep-ex/9706012

[3] T. Sjöstrand, S. Ask, J.R. Christiansen, R. Corke, N. Desai, P. Ilten, S. Mrenna, S. Prestel, C.O. Rasmussen, P.Z. Skands, Comput. Phys. Commun. 191, 159 (2015), 1410. 3012

[4] S. Chatrchyan et al. (CMS), JINST 3, S08004 (2008)

[5] M. Cacciari, G.P. Salam, G. Soyez, JHEP 04, 063 (2008), 0802 . 1189

[6] CMS Collaboration, CMS-PAS-SMP-14-015 (2015)

[7] CMS Collaboration, CMS-PAS-SMP-15-003 (2016)

[8] S. Chatrchyan et al. (CMS), Eur. Phys. J. C74, 2901 (2014), 1311.5815

[9] J. Alwall, M. Herquet, F. Maltoni, O. Mattelaer, T. Stelzer, JHEP 06, 128 (2011), 1106.0522

[10] P. Nason, JHEP 11, 040 (2004), hep-ph/0409146

[11] S. Frixione, P. Nason, C. Oleari, JHEP 11, 070 (2007), 0709. 2092

[12] S. Alioli, P. Nason, C. Oleari, E. Re, JHEP 06, 043 (2010), 1002 . 2581 